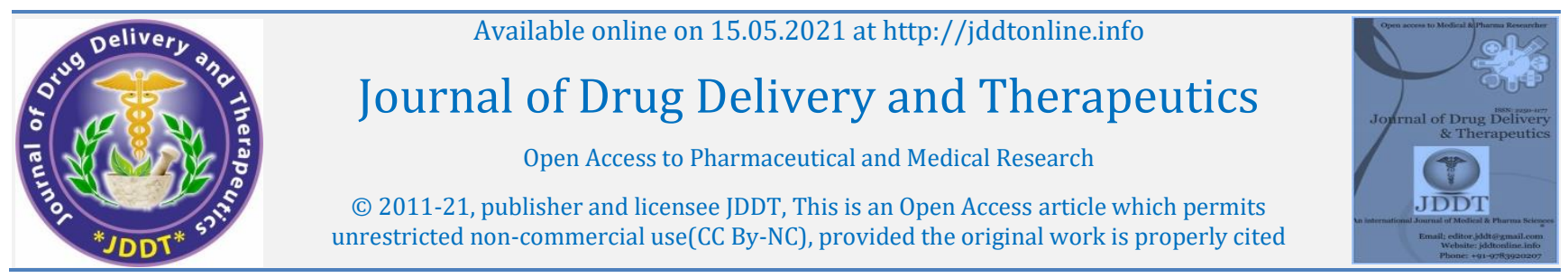

Open Access Full Text Article

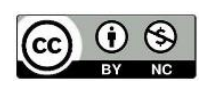

Review Article

\title{
Physiological changes during pregnancy: a mimic of medical disease and risk to existing ones
}

Kamel El-Reshaid*

Department of Medicine, Faculty of Medicine, Kuwait University, P O Box 24923, 13110 Safat, Kuwait

\section{Article Info:}

Received 21 March 2021;
Accepted 20 April 2021;
Available online 15 May 2021

*Address for Correspondence:

Kamel El-Reshaid, Department of Medicine, Faculty of Medicine, Kuwait University, P O Box 24923, 13110 Safat, Kuwait

\author{
Abstract
}

During pregnancy; multiple physiological adaptations are encountered. Their aim is to protect and nurture the developing fetus and prepare the mother for labor and delivery. They are mediated by an orchestra of maternal and placental hormones. Some of these changes influence normal biochemical values while others may mimic symptoms of medical disease. It is important to differentiate between normal physiological changes and disease pathology. The present review summarizes the changes in different systems of the body and its metabolism in an attempt to assist clinicians caring for pregnant women during health and disease.

Keywords: adaptations, changes, hormones, metabolism, physiology, pregnancy.

Cite this article as:

El-Reshaid K, Physiological changes during pregnancy: a mimic of medical disease and risk to existing ones, Journal of Drug Delivery and Therapeutics. 2021; 11(3):103-105 DOI: http://dx.doi.org/10.22270/jddt.v11i3.4708

\section{Introduction}

Physiological changes occur in pregnancy to nurture the developing fetus and prepare the mother for labor and delivery. Some of these changes influence normal biochemical values while others may mimic symptoms of medical disease. It is important to differentiate between normal physiological changes and disease pathology ${ }^{1}$. The underlying mechanism is maternal and placental hormones ${ }^{2}$.

\section{Adaptive changes during pregnancy}

1- Estrogen and progesterone maintain endometrium during menstrual cycle by follicular cells then luteal cells. To prevent its shedding; human chorionic gonadotropin (hCG) is produced from fertilized ovum to maintain corpus leutem (basis of pregnancy test) for first 5 weeks. Subsequently, the newly formed placenta secretes hCG, estrogen and progesterone. Estrogen for uterine and endometrial growth as well as for fetal organs. It also essential for growth of breast tissue and milk ducts. Progesterone is essential for placental vasculature and inhibits uterine contractures ${ }^{3}$. The increasing levels of hCG during first trimester are responsible for morning sickness \& frequent urination in early pregnancy. It will continue since it is essential suppress immunity to prevent fetal rejection: higher cold \& flue.

2- During pregnancy: (a) FSH is suppressed by high estrogen to prevent formation of more ova otherwise twins if remaining high, (b) LH is suppressed by high progesterone. If high: suspect polycystic ovarian syndrome. (c)_Relaxin relaxes muscles including uterus and ligaments for growing fetus. Moreover, it increases blood flow to kidneys and placenta.

3- Delivery: is started by increase fetal secretion of ACTH leading to high cortisol that decreases placental estrogen and progesterone and increase prostaglandin E \& F which starts uterine contractions. Cervical stretch receptors stimulate oxytocin release from hypothalamus to pituitary leading to increase uterine contractions and further stimulate more prostaglandins E \& F. Similar events are seen with any stress to fetus viz. prepartum hemorrhage or cervical disease leading to premature labor.

4- Prolactin release from pituitary increase for milk production from mammary duct in the breasts.

\section{Main maternal complaints:}

1- Weight gain

2- LL oedema

3- Nausea early pregnancy + Higher taste \& prefer salty/sour (metallic taste).

4- Falls (lax ligaments \& joints)

5- Higher temp: need more fluid \& easily dehydrated (high metabolic rate). To avoid hurting fetus: increase blood flow to skin via increase loss of fluid to cool it: dehydration. So exercise: indoor/pool \& with loose/light clothes 
6- Palpitations due to tachycardia (20\%) and syncope due to low BP \& decrease venous return by pressure on inferior vena cava: so lye on side).

7- Early in pregnancy: an increase tidal volume by $50 \%$ more than tachypnea (air hunger) \& respiratory alkalosis. However, later in pregnancy or during sleep: the diaphragm is splinted leading to shortness of breath. The need for more oxygen is for the higher metabolic rate to satisfy the demands of the growing fetus which peaks from $15^{\text {th }}$ week till delivery. If not met spontaneous hypoglycemia develops.

\section{Hematological changes:}

1. In normal pregnancy; plasma volume increases progressively by 4-6 liters till 34 weeks. Hence; anemia in the first and second trimester which normalize only by third trimester after erythropoietin increase.

2. The platelet count tends to fall progressively during normal pregnancy. Hence, thrombocytopenic only if $<100 \times 10^{9}$ cells $/$ l.

3. Increase erythropoiesis indicates increase requirement of (a) iron by 3 fold, (b) vitamin B12 by 2 folds, and (c) foliate by 15 fold.

4. Pregnancy is a hypercoagulable state in preparation for delivery. Increase risk for venous thromboembolism is present from the first trimester and for at least 12 weeks postpartum. However, coagulation tests: PT, aPTT, thrombin time: normal ${ }^{4}$.

5. Excessive exercise diverts blood from placenta to muscles. Hence, contraindicated. However, regular one (conditioning) is acceptable.

\section{Cardiovascular changes:}

1. By eight weeks' gestation, the cardiac output ( $\mathrm{CO}$ ) has already increased by $20 \%$ due estrogen ${ }^{5}$. At third trimester, CO is $30-50 \%$ above baseline. Hence, heart rate increases $15 \%$ by $20-28$ weeks yet $<100 /$ min.

1- SVR decreases by $20 \%$ secondary to the vasodilatory effect of progesterone.

2- Systolic and diastolic blood pressure drops 10-15 mm $\mathrm{Hg}$ in the first trimester and then returns to baseline in the second half of pregnancy. Cause: VD due to progesterone then shift of blood to placenta 6 .

3- Relaxin, a peptide hormone produced placenta and luteal phase of the menstrual cycle, rise after conception to a peak at the end of the first trimester and fall to an intermediate value throughout the second and third trimester. Relaxin stimulates the formation of endothelin, which in turn mediates vasodilation of renal arteries via nitric oxide (NO) synthesis ${ }^{7}$.

4- Despite activation of the renin-angiotensinaldosterone (RAA) system in early pregnancy, a simultaneous relative resistance to angiotensin Il develops due to dilatory effect of prostacyclin ${ }^{8}$.

5- Uterine enlargement beyond 20 weeks' size can compress the inferior vena cava leading to; (a) decrease preload and dizziness on standing. Hence, left lateral position is always preferred. Vasodilation on left LL due to compression of the left iliac vein by the left iliac artery and the ovarian artery.

6- No change in pulmonary arterial pressure yet $15 \%$ decrease oncotic pressure due to low albumin makes them $30 \%$ more susceptible for pulmonary oedema if fluid overload or toxemia.

7- At delivery; an increase in cardiac output since uterine contractions leads to an auto-transfusion of 300-500 $\mathrm{ml}$ of blood back into the circulation during labor. Moreover $60 \%$ increase in cardiac output due to (a) relive of IVC pressure from gravid uterus increases venous return and (b) reabsorption of oedema fluid after labor. Hence, risk for pulmonary oedema is high: (a) during second trimester and (b) immediate postpartum.

8- Physiological changes may include a bounding or collapsing pulse and an ejection systolic murmur, present in over $90 \%$ of pregnant women. The murmur may be loud and audible all over the precordium, with the first heart sound loud and possibly sometimes a third heart sound.

9- Heart pushed upwards \& to left: ECG: as if old inferior MI: Q wave (small) and inverted T wave in lead III \& LAD.

10- The risk of left ventricular failure with myocardial dysfunction or valvular disease is high during pregnancy and can manifest starting from the second trimester.

\section{Nephrourological changes:}

1- Intravascular compartment is less (placental shift) \& blood pressure low. However, renal perfusion is maintained due placental relaxin synthesis leading to endothelin formation and subsequent prostaglandin release which peaks in first then moderate in $2^{\text {nd }} \& 3^{\text {rd }}$. Hence, renal plasma flow \& GFR is increased by $50 \%{ }^{7}$.

2- Hydronephrosis due to progesterone. Changes due to smooth muscle relaxation due to progesterone. $80 \%$ of women have right physiological hydronephrosis more due to right ureter crossing the iliac and ovarian vessels at an angle before entering the pelvis. Urinary stasis in the dilated collecting system predisposes pregnant women with asymptomatic bacteriuria to pyelonephritis.

3- Due to high GFR; the reabsorption of glucose in the proximal and collecting tubule is less effective and hence glycosuria and low uric acid levels. The latter correlates well with GFR and is being used as a sensitive marker for early pre-eclampsia.

\section{Respiratory changes}

1- There is a significant increase in oxygen demand during normal pregnancy. This is due to a $15 \%$ increase in the metabolic rate and a $20 \%$ increased consumption of oxygen.

2- There is a $40-50 \%$ increase in minute ventilation, mostly due to an increase in tidal volume, rather than in the respiratory rate. This maternal hyperventilation causes arterial pO2 to increase and arterial $\mathrm{pCO}_{2}$ to fall, with a compensatory fall in serum bicarbonate to 18$22 \mathrm{mmol} / \mathrm{l}(\mathrm{pH}$ 7.44: compensated respiratory alkalosis)

\section{Gastrointestinal changes:}

1- Nausea and vomiting are very common (90\%) during pregnancy due to the increasing levels of human chorionic gonadotropin (hCG), estrogen and progesterone. 
2- The nausea symptoms usually resolve by week 20 except for hyperemesis gravidarum in $3 \%$ with potential for dehydration, electrolyte imbalance, ketonuria, weight loss and vitamin or mineral deficiencies. Prior to dextrose supplementation thiamin (vitamin B6) supplementation is essential to avoid the development of Wernicke's encephalopathy.

3- Gastropariesis is common due to progesterone.

\section{Endocrinal changes:}

1- No significant changes in thyroid.

2- In the adrenals; physiological hypercortisolism leads to striae, facial plethora, and impaired glucose tolerance.

3- Prolactin increase is due to the increasing serum oestradiol concentrations during pregnancy and is 10 times at labor for breast milk.

4- Levels of follicle-stimulating hormone (FSH) and luteinizing hormone (LH) are undetectable during pregnancy due to the negative feedback from elevated levels of estrogen, progesterone and inhibin.

5- Oxytocin levels increase in pregnancy and peak at term.

6- Levels of antidiuretic hormone (ADH) remain unchanged due to re-set of osmoreceptors for ADH by relaxin. Excessive water intake may lead to hyponatremia.

7- Gestational DM is due to maternal insulin resistance that begins in the second trimester and peaks in the third trimester. It is due to diabetogenic hormones such as human placental lactogen, growth hormone, progesterone, cortisol and prolactin. The mechanism of insulin resistance is a decrease in insulin sensitivity in the peripheral tissues such as adipocytes and skeletal muscle by interfering with insulin receptor signaling ${ }^{9}$.

\section{Changes in metabolism:}

A- Lipid:

1- The placenta produces human placental lactogen (hPL) which stimulates maternal lipolysis and fatty acid metabolism.

2- There is an increase in total serum cholesterol and triglyceride levels in pregnancy. The increase in triglyceride levels is mainly as a result of increased synthesis by the liver and decreased lipoprotein lipase activity, resulting in decreased catabolism of adipose tissue.

3- Changes in lipid metabolism accommodate the needs of the developing fetus. Increased triglyceride levels provide for the mother's energy needs while glucose is spared for the fetus. The increase in LDL cholesterol is important for placental steroidogenesis.

B- Protein:

Pregnant women require an increased intake of protein during pregnancy. Amino acids are actively transported across the placenta to fulfill the needs of the developing fetus.

C- Calcium:

1- fetal needs are met by increased intestinal absorption, which doubles from 12 weeks' gestation due to activation of 25 dihydroxy vitamin $\mathrm{D}$ and is associated with hypercalcuria.

2- However the peak fetal demand for calcium is only in the third trimester. This early increase in calcium absorption may allow the maternal skeleton to store calcium in advance. Hence, if intake is excessive; kidney stones will form 10 .

D- Pregnancy and osteoporosis:

1- Calcium is stored and not used till $3^{\text {rd }}$ trimester. Hence no osteoporosis due to pregnancy.

2- However, there is increased lordosis and joint laxity.

\section{References:}

1- Clinical biochemistry of pregnancy. Lockitch G. Crit Rev Clin Lab Sci. 1997; 34: 67-139.

2- Hayes, Meghan; Larson, Lucia (2012). Chapter 220. Overview of physiologic changes of pregnancy. Principles and Practice of Hospital Medicine. The McGraw-Hill Companies. ISBN 9780071603898.

3- Guyton and hall (2005). Textbook of Medical Physiology (11 ${ }^{\text {th }}$ ed.). Philadelphia: Saunders. pp. 103g.

4- Medicine. The McGraw-Hill Companies. ISBN 9780071603898.

5- Rodger M, Sheppard D, Gandara E, Tinmouth A. Haematological problems in obstetrics. Best Prac Res Clin Obstet Gynaecol. 2015; 29: 671-684.

6- Wilson M, Morganti AA, Zervoudakis I, Letcher RL, Romney BM, Von Oeyon P. et al. Blood pressure, the renin-aldosterone system and sex steroids throughout normal pregnancy. Am J Med. 1980; 68: 97-104.

7- Conrad KP. Emerging role of relaxin in the maternal adaptations to normal pregnancy: implications for preeclampsia. Semin Nephrol. 2011; 31:15-32.

8- Irani RA, Xia Y. Renin angiotensin signaling in normal pregnancy and preeclampsia. Semin Nephrol. 2011; 31: 47 58.

9- Mazaki-Tovi S, Kanety H, Pariente C, Hemi R, Yissachar E, Schiff E, Cohen O, Sivan E. Insulin sensitivity in late gestation and early postpartum period: the role of circulating maternal adipokines. Gynecol Endocrinol. 2011; 27: 725-731.

10- Kovacs CS. Calcium and phosphate metabolism and related disorders during pregnancy and lactation. In: Feingold KR, Anawalt B, Boyce A, et al., editors. Endotext [Internet]. South Dartmouth (MA): MD Text.com. NCBI Bookshelf (2018 update). 